\title{
Examination of Possible Flow Turbulence during Flow-Mediated Dilation Testing
}

\author{
Lee Stoner ${ }^{1,2^{*}}$, Manning Joseph Sabatier ${ }^{1}$, Joanna Mary Young ${ }^{3}$ \\ ${ }^{1}$ Lipid and Diabetes Research Group, Diabetes Research Institute, Christchurch, New Zealand \\ ${ }^{2}$ Department of Kinesiology, University of Georgia, Athens, USA \\ ${ }^{3}$ Division of Biological Sciences, Clayton State University, Morrow, USA \\ E-mail: *dr.l.stoner@gmail.com \\ Received August 31, 2011; revised September 8, 2011; accepted September 14, 2011
}

\begin{abstract}
The validity of the flow-mediated dilation (FMD) test has been doubted due to the lack of normalization to the primary stimulus, shear stress. Shear stress can be calculated using a simplified mathematical model based on Poiseuille's law. Poiseuille's law assumes that the blood velocity profile is parabolic. The presence of turbulence will violate this assumption. The Reynolds number (RE) is used to define critical values for the transition from laminar to turbulent flow. Between RE values of 2000 and 4000, flow enters a transitional phase where turbulence is possible. Purpose: To determine whether brachial artery blood flow becomes turbulent during reactive hyperemia following forearm ischemia. Methods: Eleven healthy male subjects (25 \pm 5 years) were tested. Brachial artery diameters and blood velocities were measured continuously following 2, 4, 6 and 10 minutes ischemia. The peak post-ischemic $\mathrm{RE}$ ( $\mathrm{RE}_{\text {peak }}$ ) and $\mathrm{RE}$ integrated over 40 seconds $\left(\mathrm{RE}_{40}\right)$ post-ischemia were calculated. Results: There was a significant change in $\mathrm{RE}_{\text {peak }}\left(\mathrm{F}_{4,7}=98.573, \mathrm{p}=\leq\right.$ $0.001)$ and $\mathrm{RE}_{40}\left(\mathrm{~F}_{4,7}=50.613, \mathrm{p}=\leq 0.001\right)$ in response to ischemia. Within-subjects contrasts revealed a significant increase in $\mathrm{RE}_{\text {peak }}$ and $\mathrm{RE}_{40}$ for each duration of ischemia versus baseline $(\mathrm{p}=\leq 0.001)$. Following 4 minutes of ischemia there was approximately 12 seconds of potentially turbulent flow. Conclusion: Blood flow transitions between laminar and turbulent flow during ischemia-induced reactive hyperemia. This may limit the efficacy of estimating shear stress when using the standard FMD test protocol.
\end{abstract}

Keywords: Flow Mediated Dilatation, Blood Flow, Shear Stress, Turbulence, Reynolds Number, Poiseuille's Law

\section{Introduction}

Disruption of the endothelium lining the conduit arteries is thought to occur early in the pathogenesis of cardiovascular disease [1]. Flow-mediated dilation (FMD) has emerged as the non-invasive standard for assessing in vivo endothelial function [2]. Reduced FMD is an early marker of atherosclerosis [2] and has been noted for its predictive capacity for future cardiovascular complications $[3,4]$. This test, typically conducted on the brachial artery, is accomplished by inflating a tourniquet around the forearm to a supra-systolic pressure for 4 - 5 minutes [2-5] - though longer durations have also been used [6-8]. Release of the tourniquet results in a large transient increase in shear stress upstream in the brachial artery. The magnitude of FMD, typically expressed as the percentage increase in diameter above rest, is used to represent endothelial health.
The validity of the FMD test has been doubted due to the lack of normalization to the primary stimulus, shear stress [9-13]. Clinical studies in humans, including FMD studies, typically estimate shear stress by employing a simplified mathematical model based on Poiseuille's law, as shown in Equation (1):

$$
\text { Shear Rate }(\gamma)=\frac{2(2+n) v}{d}
$$

where $d$ is the internal arterial diameter, $v$ is time averaged mean blood velocity, and $n$ represents the shape of the velocity profile. (For a fully developed parabolic profile, $n$ is 2.)

Poiseuille's law assumes that: 1) The fluid (blood) is Newtonian. 2) Blood flows through a rigid tube. 3) Whole blood viscosity represents viscosity at the vessel wall and is linearly proportional to shear rate. And, 4) The velocity profile is parabolic. First, although blood is 
non-Newtonian, the effect of the non-Newtonian behaveior does not appear to be pronounced in large arteries [14]. Second, blood vessels are distensible, meaning that wall shear rate may be $\sim 30 \%$ less in a distensible artery as compared with a rigid tube [14]. Third, blood viscosity exhibits low intra-subject variability [16], particularly among a healthy, homogeneous group. Fourth, in arteries, the velocity profile will generally not develop to a full parabola as a consequence of flow unsteadiness and short vessel entrance lengths. However, in the brachial artery, under resting conditions, the underestimation is less pronounced-likely due to a more parabolic velocity profile in this artery, i.e., $n$ (velocity profile) is closer to 2 [17]. However, this may only be true for resting conditions; the possibility of flow turbulence during reactive hyperemia has not been ascertained.

The Reynolds number (RE) is a dimensionless number used to characterize laminar or turbulent flow. The Reynolds number has long been used to define critical values for the transition from laminar to turbulent flow [18-19]. Laminar flow occurs when RE $<2000$ and turbulent flow occurs when $\mathrm{RE}>$ 4000. Between 2000 and 4000, flow enters a transitional phase where turbulence is possible, dependent on vessel characteristics.

The purpose of this study was to determine whether brachial artery blood flow becomes turbulent during reactive hyperemia following forearm ischemia.

\section{Materials and Methods}

\subsection{Subjects and Study Design}

Thirteen healthy, physically active, young male subjects were recruited. None of the subjects reported cardiovascular disease complications. The study was approved by the University of Georgia Institutional Review Board. Written informed consent was obtained from the subjects after they were given a detailed description of the procedures.

The test sessions were performed between the hours of 7 am and 10 am to reduce circadian variation. All subjects were asked to report to the laboratory in the fasted condition, having refrained from exercise for 48 hours prior to testing. Subjects were also asked not to consume caffeine or administer any medications with known vascular actions prior to testing. Subjects were excluded from the study if they were currently prescribed medications with known vascular effects. All stages of testing were performed in a climate controlled laboratory setting.

\subsection{Protocol}

Testing commenced following at least 20 minutes of quiet supine rest. Left arm brachial artery diameters and blood velocities were measured continuously following 2 minutes, 4 minutes, 6 minutes and 10 minutes ischemia. Following each ischemic period there was 5 minutes recovery. This duration has previously been demonstrated to allow arterial diameter and hemodynamic conditions to return to baseline in health young subjects [20]. Several studies have reported that repeated FMD testing does not affect FMD magnitude [21-23].

\subsection{Ischemia}

A pneumatic tourniquet (Hokanson, Inc., Seattle, Washington) placed around the limb, distal to the insonated artery, was rapidly inflated (1 seconds - 2 seconds) to a pressure of approximately $100 \mathrm{mmHg}$ above the systolic blood pressure. Blood pressure measurements were taken on the non-imaged arm.

\subsection{Ultrasound Diameter Measurements}

High-resolution Brightness-mode (B-mode) ultrasound measurements were made using a GE 400CL duplex color Doppler unit (GE Medical, Milwaukee, Wisconsin) equipped with a $7 \mathrm{MHz}-13 \mathrm{MHz}$ linear array transducer (LA39). The brachial artery of the left arm was measured in the distal third of upper arm. Care was taken to ensure that the vessel clearly extended across the entire [un-zoomed] imaging plane to minimize the likelihood of skewing the vessel walls. Magnification and focal zone settings were then adjusted to optimize imaging of the proximal and distal vessel walls. The image was comprised of $400 \times 400$ pixels over an area of $16 \times 16 \mathrm{~mm}$, with a pixel resolution of $0.04 \times 0.04 \mathrm{~mm}$. A specialized probe holding device enabled precise positioning and ensured that pressure on the artery was minimized. The precise position of the ultrasound probe was recorded and marked. Ultrasound global (acoustic output, gain, dynamic range, gamma, and rejection) and probe-dependent (zoom factor, edge enhancement, frame averageing, and target frame rate) settings were standardized.

\subsection{Diameter Analysis}

Moving Picture Experts Group-2 (MPEG-2) recordings were captured using a Dell Laptop PC equipped with a video capture device (ADS technologies, Cerritos, California). Video files collected at 30 frames/second were converted to Joint Photographic Experts Group (JEPG) images and subsequently used to make 30 diameter measurements/second. JPEG images provide comparable accuracy for ultrasound image measurements compared to the Digital Image and Communications in Medicine 
(DICOM) standard [24]. Diameters were measured continuously during the transient conditions. Images were measured offline using semi-automated edge-detection software custom written to interface with the LabVIEW data acquisition platform (version 8.1, National Instruments, Austin, Texas) [6,25]. Custom written Excel Visual Basic code was used to fit peaks and troughs to diameter waveforms in order to calculate diastolic, systolic, and mean diameters. Mean diameters were used for analysis. A recent study indicates that calculating FMD based on mean diameters yields comparable results to calculations based on end-diastolic diameters [26]. The within-session $\mathrm{SEM}_{3,1}$ for diameter measurement with the described set-up is $0.046 \mathrm{~mm}$. Between-day coefficients of variation are $2.7 \%$ resting diameter measurements and $2.4 \%$ for peak diameter measurements following reactive hyperemia [27].

\subsection{Blood Velocities}

Sonication angle was kept constant between $45^{\circ}-65^{\circ}$ and the sample volume included most of the vessel. Blood velocities were calculated using the advanced vascular package supplied with the GE 400CL ultrasound machine. A custom written Optical Character Reading (OCR) package written in LabVIEW (version 6.1) was used to capture blood velocities for each cardiac cycle. When more than two values were recorded for a given second, the average was computed. Obvious outliers were removed and missing values were replaced using linear interpolation. In our laboratory, between-day coefficients of variation for peak velocity measurements are $14.6 \%$ [27].

\subsection{Shear Rate}

The 30 diameter measurements/second were aggregated to $1 /$ second and synchronized with blood velocities. Shear rates were calculated using Equation (2):

$$
\text { Shear Rate }\left(s^{-1}\right)=8 v / d
$$

where $d$ is the internal arterial diameter and $v$ is time averaged mean blood velocity. Time averaged maximum blood velocities were used to calculate shear rate. Time averaged maximum velocity is the average of the highest velocities throughout the cardiac cycle. Our ultrasound machine more reliably calculates these velocities when compared to time averaged mean blood velocities [28].

The shear response was integrated over 40 seconds post-ischemia as previously reported [29]. Previous studies have shown that the integrated shear response is primary stimulus, not the peak shear response. Shear rate has been used as a surrogate measure of shear stress in a number of previous studies [16,22,30-32].

\subsection{Reynold's Number}

Reynold's number was calculated second-by-second using Equation (2):

$$
\operatorname{Re}_{40}=v d p / \eta
$$

where $v=$ peak systolic velocity, $d=$ diameter, $\rho=$ density, and $\eta=$ viscosity. Density was assumed to be 1060 $\mathrm{kg} / \mathrm{m}^{3}$, and viscosity $0.0035 \mathrm{~Pa} \cdot \mathrm{s}$.

The peak post-ischemic RE ( $\left.\mathrm{RE}_{\text {peak }}\right)$, as well as RE integrated over 40 seconds $\left(\mathrm{RE}_{40}\right)$ post-ischemia was calculated.

\subsection{Blood Pressures}

Blood pressures were continuously measured on the right (non-imaged) arm using a finger photo-plethysmographic device (Finapress 2300, Ohmeda, Englewood, Colorado) interfaced to a Biopac data acquisition system (MP100, Biopac Systems, Inc., Goleta, California). To ensure the Finapress was correctly calibrated, the systolic and diastolic blood pressure values were checked against recordings from a semi-automated blood pressure device (Datascope Accutor 3, Montvale, New Jersey). If systolic and diastolic measurements did not agree within 5 $\mathrm{mmHg}$, the finger cuff was adjusted until the two devices agreed [33].

\subsection{Statistics}

Descriptive data for each stage of testing are expressed as means $( \pm \mathrm{SD})$. One-way repeated measures analysis of variance was used to examine differences in $R_{\max }$ and the reference category. The Statistical Package for Social Sciences (SPSS Inc, Chicago, IL) 17.0 for Windows was used for data analysis. $\mathrm{P}<0.05$ was considered statistically significant.

\section{Results}

Analysis was conducted on eleven of the thirteen subjects. One subject was omitted due to poor B-mode Ultrasound image quality, and another subject due to technical difficulties. For the remaining eleven subjects the average age was 25 years +5 years, height $176 \mathrm{~cm}+6 \mathrm{~cm}$, and weight $79 \mathrm{~kg}+11 \mathrm{~kg}$. Subjects exercised an average of 5 times/week +4 times/week for 59 minutes/session +26 minutes/session. Average systolic, diastolic and mean blood pressures were $120 \mathrm{mmHg}+10 \mathrm{mmHg}, 70 \mathrm{mmHg}$ $+7 \mathrm{mmHg}$, and $89 \mathrm{mmHg}+8 \mathrm{mmHg}$, respectively. Blood pressures did not significantly differ between conditions. 


\subsection{Diameter and Shear Rate Responses}

Figure 1 shows representative examples of velocity waveforms at rest and 10 seconds post 10 minutes ischemia. At rest, the spectral waveform is characterized by a narrow range of frequencies, with the energy concentrated on the highest frequencies. Post-ischemia, a wide range of frequencies is seen, with energy spread more evenly across the range of frequencies. The spectral broadening may indicate a more parabolic flow profile, or the presence of turbulent flow.

Figure 2 shows the second-by-second diameter and shear rate responses to each duration of ischemia. Figure 3 shows mean average integrated shear rate plotted against peak diameters for each duration of ischemia. There was a large amount of variation in diameter. The majority of this variation can be explained by two factors. Firstly, standard deviations are misleading since continuous data is forced to become categorized according to stage-particularly important when considering that resting diameters ranged from $3.28 \mathrm{~mm}$ to $4.78 \mathrm{~mm}$. Secondly, the shear rate responses varied greatly between subjects; thereby contributing to the variation in diameter.

\subsection{Reynolds Number}

Table 1 characterizes the flow characteristics measured during reactive hyperemia. There was a significant change in $\mathrm{RE}_{\text {peak }}\left(\mathrm{F}_{4,7}=98.573, \mathrm{p}=\leq 0.001\right)$ and $\mathrm{RE}_{40}$ $\left(\mathrm{F}_{4,7}=50.613, \mathrm{p}=\leq 0.001\right)$ in response to ischemia. Within-subjects contrasts revealed a significant increase in $\mathrm{RE}_{\text {peak }}$ and $\mathrm{RE}_{40}$ for each duration of ischemia versus baseline. Figure 4 shows the potential for turbulence during reactive hyperemia. After 4 minutes of ischemia

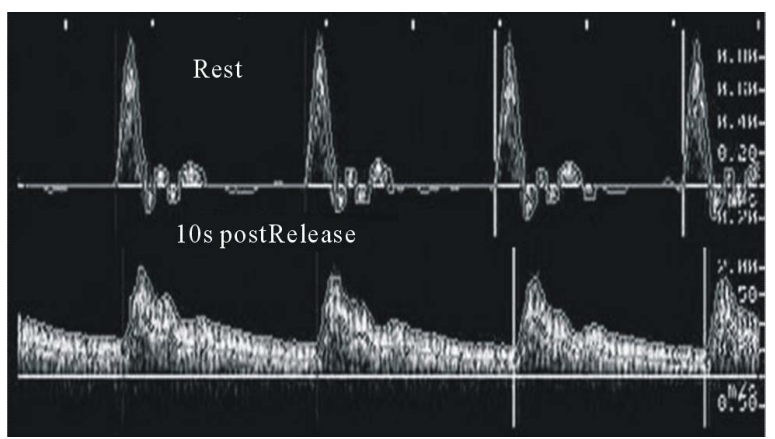

Figure 1. Representative velocity waveforms taken from one subject at rest and 10 seconds post 10 minutes ischemia. The resting waveform is characterized by a narrow range of frequencies, with the energy concentrated on the highest frequencies. Post-ischemia a wide range of frequencies is seen, with energy spread more evenly across the range of frequencies. The spectral broadening may indicate a more parabolic flow profile, or the presence of turbulent flow.

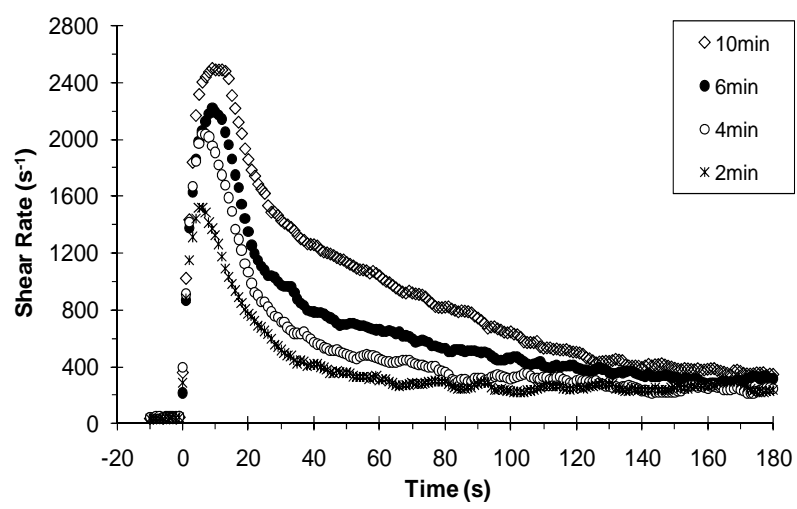

(a)

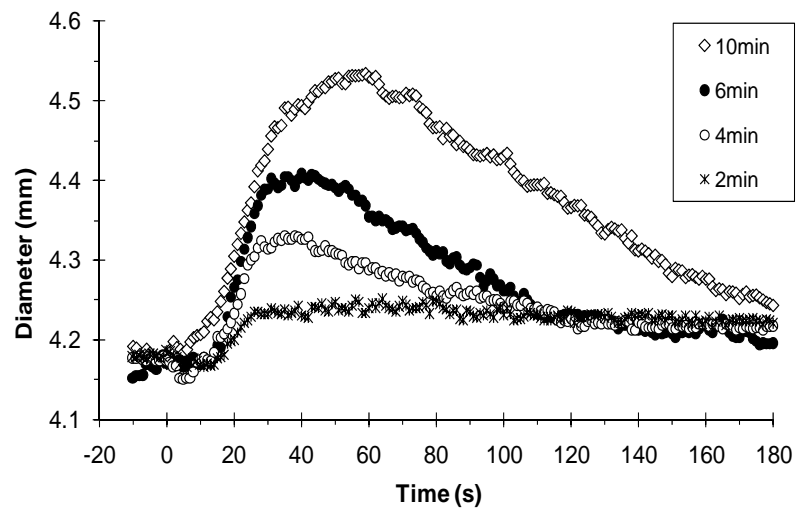

(b)

Figure 2. Mean shear rates (a) and diameters (b) post ischemia.

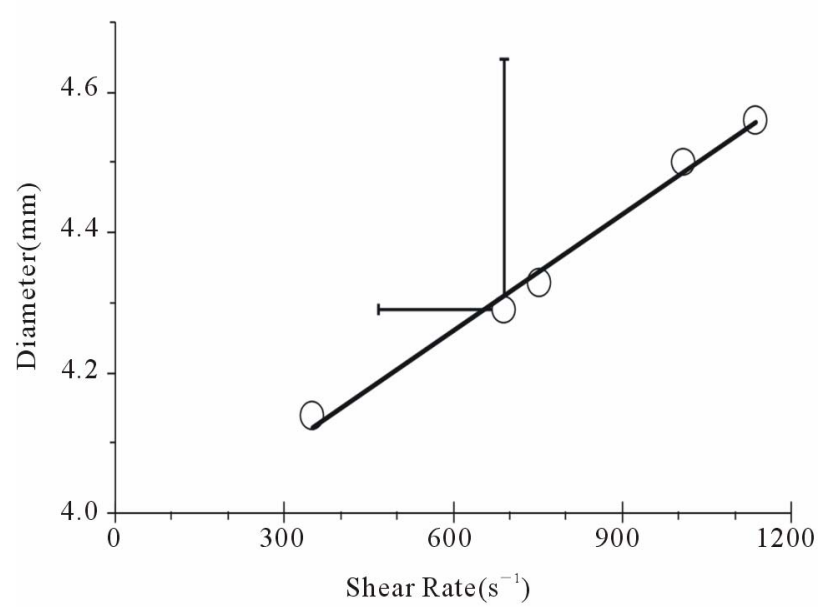

Figure 3. Mean shear rates plotted against peak diameters for transient and steady-state conditions. Shear rates are integrated over 40s post-ischemia. Error bars show representative standard deviations.

RE was above the transition threshold for turbulence for the first 12 seconds post-ischemia. RE increases with the duration of ischemia. 
Table 1. Brachial artery flow characteristics following forearm ischemia.

\begin{tabular}{|c|c|c|c|c|c|c|c|c|c|c|c|c|c|c|}
\hline \multirow{3}{*}{$\begin{array}{c}\text { Ischemia } \\
\text { min } \\
\text { Rest }\end{array}$} & \multicolumn{2}{|c|}{$\mathrm{D}_{\text {TTP }}$} & \multicolumn{2}{|c|}{$\mathrm{SR}_{\mathrm{TTP}}$} & \multicolumn{2}{|c|}{$\mathrm{SR}_{\text {peak }}$} & \multicolumn{2}{|c|}{$\mathrm{SR}_{40}$} & \multicolumn{2}{|c|}{$\mathrm{RE}_{\text {peak }}$} & \multicolumn{2}{|c|}{$\mathrm{RE}_{40}$} & \multicolumn{2}{|c|}{$\mathrm{RE}_{\text {time }}$} \\
\hline & \multicolumn{2}{|c|}{$\mathrm{s}$} & \multicolumn{2}{|c|}{$\mathrm{s}$} & \multicolumn{2}{|c|}{$\mathrm{s}^{-1}$} & \multicolumn{2}{|c|}{$\mathrm{s}^{-1}$} & \multirow[b]{2}{*}{884} & \multirow[b]{2}{*}{ (388) } & \multirow[b]{2}{*}{884} & \multirow[b]{2}{*}{ (388) } & \multicolumn{2}{|c|}{$\mathrm{s}$} \\
\hline & 0 & (0) & 0 & (0) & 231 & (98) & 231 & (98) & & & & & 0 & (0) \\
\hline 2 & 52 & (26) & 5 & (3) & 1586 & (254) & 837 & (227) & 1882 & $(239) *$ & 1501 & $(175)^{*}$ & 2 & (3) \\
\hline 4 & 35 & (9) & 8 & (3) & 2103 & (247) & 1154 & (177) & 2396 & $(398)^{*}$ & 1784 & $(188)^{*}$ & 12 & (6) \\
\hline 6 & 42 & (11) & 9 & (2) & 2299 & (408) & 1373 & (265) & 2654 & $(312)^{*}$ & 1914 & $(225)^{*}$ & 18 & (13) \\
\hline 10 & 55 & (19) & 11 & (2) & 2575 & (377) & 1781 & (310) & 2723 & $(300)^{*}$ & 2214 & $(236)^{*}$ & 36 & (16) \\
\hline
\end{tabular}

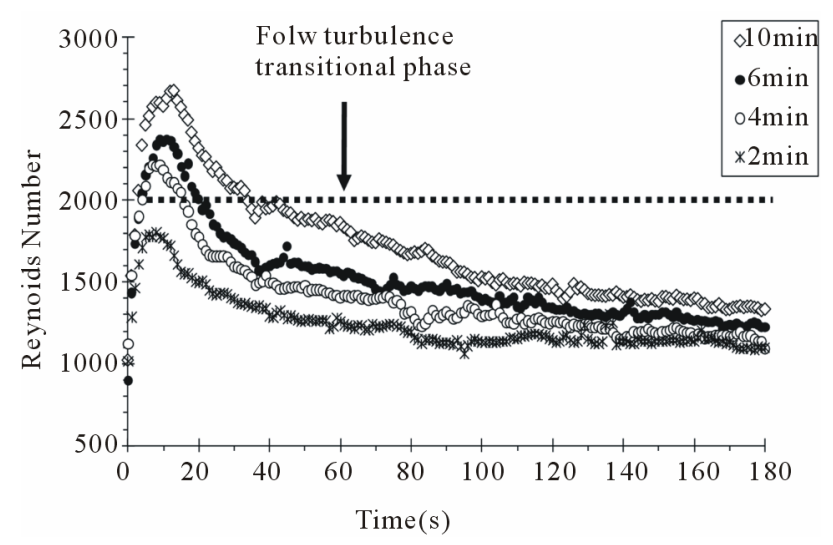

Figure 4. Change in Reynolds number during reactive hyperemia.

\section{Discussion}

This study found that increasing durations of forearm ischemia correspondingly increase the potential for flow turbulence in the brachial artery during reactive hyperemia. While no duration of ischemia increased the RE to the critical threshold for turbulence ( $R E>4000)$, even two minutes of ischemia increased the RE to the threshold for transition between laminar and turbulent flow (RE > 2000). Following 4 minutes of ischemia, a duration often used for the FMD test, there was approximately 12 seconds of potentially turbulent flow. These observations suggest that one of the fundamental assumptions for calculating shear stress based on Poiseuille's law, i.e., a parabolic velocity profile, may be violated during reactive hyperemia.

\subsection{Implications}

Despite its potential, validity of the FMD test has been doubted due to lack of normalization to the primary stimu- lus, shear stress [9-13]. In order to efficaciously compare groups of individuals the shear stimulus should be considered. For instance, Mitchell et al. [10] demonstrated that reduced FMD may be attributable not only to impaired endothelial release of dilatory molecules, but also to a smaller shear stimulus. However, the findings from the current study suggest that Poiseuille's law may not adequately characterize shear stress, at least when shear stress is estimated during reactive hyperemia.

In order to efficaciously compare shear stress estimates between groups: 1) The duration of ischemia should be shortened. Or, 2) FMD should be estimated using steady-state increases in shear stress. Shortening the duration of ischemia will not completely nullify the potential for flow turbulence and will limit the magnitude of FMD. Furthermore, limiting the magnitude of FMD will po- tentially limit the reliability of the test. Alternatively, FMD can evaluated by using sustained increases in shear stress, e.g., through local hand warming and low-intensity handgrip exercise [7,22,34,35]. Consideration has to be given to the mechanism(s) inducing FMD, since said mechanisms may be dependent on the duration of the shear stimulus [34,36-39]. Notably, FMD in response to sustained shear stress is likely less NO-dependent [40]. Nonetheless, the endothelium is still thought to primarily govern vasodilation under steady-state shear rate conditions. For instance, studies have shown that hand warming has no effect on brachial artery diameter when flow is not allowed to rise [22,30,34]. Furthermore, pharmacological blockade of the autonomic nervous system has no effect on radial artery FMD in response to hand warming [34], consistent with animal studies showing that FMD is preserved after surgical or pharmacological denervation $[41,42]$.

A recent meta-analysis by Inaba et al. [43], which was subsequently re-analyzed by Green et al. [44], assessed the CVD prognostic strength of FMD by examining the association between brachial artery FMD and future car- 
diovascular events. Green et al. found that FMD resulting from more intense and prolonged shear stimuli using proximal cuff placement, which has been demonstrated to be less NO-dependent, is a better predictor of CVD risk [40]. Further study is needed to confirm these findings and determine whether FMD in response to sustained increases in shear rate provides greater prognostic strength for detecting future CVD events.

\subsection{Limitations}

For the current study a homogenous group of healthy, male subjects were recruited in order to obtain optimal data. This aim was accomplished, however further work is required in order to determine the potential for turbulence during reactive hyperemia across different populations, particularly populations exhibiting CVD risk factors.

This study is limited by the RE itself, which was originally defined for laminar flow in a rigid pipe and not for pulsatile blood flow in compliant arteries. In addition, the calculation of RE was dependent on assumed blood viscosity and density. However, blood viscosity exhibits low intra-subject variability [16], particularly in a healthy, homogenous group, and therefore is unlikely to have affected our results.

\subsection{Summary}

One of the fundamental assumptions for calculating shear stress based on Poiseuille's law, i.e., a parabolic velocity profile, may be violated during ischemia-induced reactive hyperemia. This potentially limits the efficacy of estimating shear stress when using the standard FMD test protocol.

\section{References}

[1] R. Ross, "The Pathogenesis of Atherosclerosis: A Perspective for the 1990s," Nature, Vol. 362, No. 6423, 1993, pp. 801-809. doi:10.1038/362801a0

[2] D. S. Celermajer, et al., "Non-Invasive Detection of Endothelial Dysfunction in Children and Adults at Risk of Atherosclerosis," Lancet, Vol. 340, No. 8828, 1992, pp. 1111-1115. doi:10.1016/0140-6736(92)93147-F

[3] T. Yoshida, et al., "Prognostic Value of Flow-Mediated Dilation of the Brachial Artery in Patients with Cardiovascular Disease," Internal Medicine, Vol. 45, No. 9, 2006, pp. 575-579. doi:10.2169/internalmedicine.45.1534

[4] J. Grewal, et al., "Assessment of Novel Risk Factors in Patients at Low Risk for Cardiovascular Events Based on Framingham Risk Stratification," Clinical \& Investigative Medicine, Vol. 26, No. 4, 2003, pp. 158-165.

[5] M. C. Corretti, et al., "Guidelines for the Ultrasound Assessment of Endothelial-Dependent Flow-Mediated Vasodilation of the Brachial Artery: A Report of the In- ternational Brachial Artery Reactivity Task Force," Journal of the American College of Cardiology, Vol. 39, No., 2002, pp. 257-265. doi:10.1016/S0735-1097(01)01746-6

[6] M. J. Sabatier, et al., "Doppler Ultrasound Assessment of Posterior Tibial Artery Size in Humans," Journal of Clinical Ultrasound, Vol. 34, No. 5, 2006, pp. 223-230. doi: $10.1002 /$ jcu.20229

[7] L. Stoner, et al., "Relationship between Blood Velocity and Conduit Artery Diameter and the Effects of Smoking on Vascular Responsiveness," Journal of Applied Physiology, Vol. 96, No. 6, 2004, pp. 2139-2145. doi:10.1152/japplphysiol.01107.2003

[8] C. D. Black, B. Vickerson and K. K. McCully, "Noninvasive Assessment of Vascular Function in the Posterior Tibial Artery of Healthy Humans," Dynamic Medicine, Vol. 2, No. 1, 2003, p. 1. doi:10.1186/1476-5918-2-1

[9] K. E. Pyke and M. E. Tschakovsky, "The Relationship between Shear Stress and Flow-Mediated Dilatation: Implications for the Assessment of Endothelial Function," The Journal of Physiology, Vol. 568, No. 2, 2005, pp. 357-369. doi:10.1113/jphysiol.2005.089755

[10] G. F. Mitchell, et al., "Local Shear Stress and Brachial Artery Flow-Mediated Dilation: The Framingham Heart Study," Hypertension, Vol. 44, No. 2, 2004, pp. 134-139. doi:10.1161/01.HYP.0000137305.77635.68

[11] B. A. Parker, T. L. Trehearn and J. R. Meendering, "Pick Your Poiseuille: Normalizing the Shear Stimulus in Studies of Flow-Mediated Dilation," Journal of Applied Physiology, Vol. 107, No. 4, 2009, pp. 1357-9. doi:10.1152/japplphysiol.91302.2009

[12] L. Stoner and K. McCully, "Blood Velocity Parameters that Contibute to Flow-Mediated Dilation," LAP LAMBERT Academic Publishing, Saarbrücken, 2011.

[13] A. Koller, D. Sun and G. Kaley, "Role of Shear Stress and Endothelial Prostaglandins in Flow- and Viscosity-Induced Dilation of Arterioles in vitro," Circulation Research, Vol. 72, No. 6, 1993, pp. 1276-1284.

[14] S. Chien, et al., "Effects of Hematocrit and Plasma Proteins on Human Blood Rheology at Low Shear Rates," Journal of Applied Physiology, Vol. 21, No. 1, 1966, pp. 81-87.

[15] D. D. Duncan, et al., "The Effect of Compliance on Wall Shear in Casts of a Human Aortic Bifurcation," Journal of Biomechanical Engineering, Vol. 112, No. 2, 1990, pp. 183-8. doi:10.1161/01.HYP.0000137305.77635.68

[16] A. Gnasso, et al., "Association between intima-Media Thickness and Wall Shear Stress in Common Carotid Arteries in Healthy Male Subjects," Circulation, Vol. 94, No. 12, 1996, pp. 3257-3262.

[17] R. Dammers, et al., "Shear Stress Depends on Vascular Territory: Comparison between Common Carotid and Brachial Artery," Journal of Applied Physiology, Vol. 94, No. 2, 2003, pp. 485-489.

[18] O. Reynolds, "An Experimental Investigation of the Circumstances Which Determine Whether the Motion of Water Shall be Direct or Sinuous, and of the Law of Re- 
sistance in Parallel Channels," Philosophical Transactions of the Royal Society B: Biological Sciences, Vol. 174, 1883, pp. 935-982.

[19] N. Westerhof, N. Stergiopulos and M. I. M. Noble, "Snapshots of Hemodynamics," Springer, New York, 2005.

[20] L. Stoner, et al., "Occasional Cigarette Smoking Chronically Affects Arterial Function," Ultrasound in Medicine \& Biology, Vol. 34, No. 12, 2008, pp. 1885-1892. doi:10.1161/01.HYP.0000137305.77635.68

[21] K. E. Pyke and F. Jazuli, "Impact of Repeated Increases in Shear Stress via Reactive Hyperemia and Handgrip Exercise: No Evidence of Systematic Changes in Brachial Artery FMD," American Journal of Physiology - Heart and Circulatory Physiology, Vol. 300, No. 3, 2011, pp. H1078-1089. doi:10.1152/ajpheart.00736.2010

[22] K. E. Pyke, E. M. Dwyer and M. E. Tschakovsky, "Impact of Controlling Shear Rate on Flow-Mediated Dilation Responses in the Brachial Artery of Humans," Journal of Applied Physiology, Vol. 97, No. 2, 2004, pp. 499-508. doi:10.1152/japplphysiol.01245.2003

[23] R. A. Harris, et al., "Variability of Flow-Mediated Dilation Measurements with Repetitive Reactive Hyperemia," Vascular Medicine, Vol. 11, No. 1, 2006, pp. 1-6. doi:10.1191/1358863x06vm641oa

[24] N. J. Hangiandreou, et al., "The Effects of Irreversible JPEG Compression on an Automated Algorithm for Measuring Carotid Artery Intima-Media Thickness from Ultrasound Images," Journal of Digital Imaging, Vol. 15, No. S1, 2002, pp. 258-260.

[25] L. Stoner, et al., "Upper versus Lower Extremity Arterial Function after Spinal Cord Injury," Journal of Spinal Cord Medicine, Vol. 29, No. 2, 2006, pp. 138-146.

[26] T. J. Kizhakekuttu, et al., "Measuring FMD in the brachial artery: how important is QRS gating?" Journal of Applied Physiology, Vol. 109, No. 4, 2010, pp. 959-65. doi:10.1152/japplphysiol.00532.2010

[27] L. Stoner, et al., "The Relationship between Blood Velocity and Conduit Artery Diameter, and the Effects of Smoking on Vascular Responsiveness," Journal of Applied Physiology, Vol. 96, No. 9, 2004, pp. 2139-2145.

[28] J. L. Olive, et al., "Blood Flow and Muscle Fatigue in SCI Individuals during Electrical Stimulation," Journal of Applied Physiology, Vol. 94, No. 2, 2003, pp. 701-8.

[29] L. Stoner and K. McCully, "Peak- and Time-Integrated Shear Rates Independently Predict Flow-Mediated Dilation," Journal of Clinical Ultrasound, 2011, In Press.

[30] R. Joannides, et al., "Evaluation of the Determinants of Flow-Mediated Radial Artery Vasodilatation in $\mathrm{Hu}-$ mans," Clinical and Experimental Hypertension, Vol. 19, No. 5-6, 1997, pp. 813-826.

[31] A. C. Betik, V. B. Luckham and R. L. Hughson, "Flowmediated Dilation in Human Brachial Artery after Different Circulatory Occlusion Conditions," American Journal of Physiology - Heart and Circulatory Physiology, Vol. 286, No. 1, 2004, pp. H442-H448. doi:10.1152/ajpheart.00314.2003
[32] J. Padilla, et al., "Normalization of Flow-Mediated Dilation to Shear Stress Area under the Curve Eliminates the Impact of Variable Hyperemic Stimulus," Cardiovascular Ultrasound, Vol. 6, 2008, p. 44. doi:10.1186/1476-7120-6-44

[33] B. Silke and D. McAuley, "Accuracy and Precision of Blood Pressure Determination with the Finapres: An Overview Using Re-sampling Statistics," Journal of Human Hypertension, Vol. 12, No. 6, 1998, pp. 403-9. doi:10.1038/sj.jhh.1000600

[34] M. J. Mullen, et al., "Heterogenous Nature of Flow- Mediated Dilatation in Human Conduit Arteries in vivo: Relevance to Endothelial Dysfunction in Hypercholesterolemia," Circulation Research, Vol. 88, No. 2, 2001, pp. 145-151.

[35] R. Joannides, et al., "Influence of Vascular Dimension on Gender Difference in Flow-Dependent Dilatation of Peripheral Conduit Arteries," American Journal of Physiology - Heart and Circulatory Physiology, Vol. 282, No. 4, 2002, pp. H1262-H1269. doi:10.1152/ajpheart.00209.2001

[36] J. A. Frangos, et al., "Flow Effects on Prostacyclin Production by Cultured Human Endothelial Cells," Science, Vol. 227, No. 4693, 1985, pp. 1477-1479. $10.1126 /$ science. 3883488

[37] M. J. Kuchan, H. Jo and J. A. Frangos, "Role of G Proteins in Shear Stress-Mediated Nitric Oxide Production by Endothelial Cells," American Journal of Physiology, Vol. 267, No. 3, 1994, pp. C753-C758.

[38] J. A. Frangos, T. Y. Huang and C. B. Clark, "Steady Shear and Step Changes in Shear Stimulate Endothelium via Independent Mechanisms--Superposition of Transient and Sustained Nitric Oxide Production," Biochemical and Biophysical Research Communications, Vol. 224, No. 3, 1996, pp. 660-665.

doi:10.1006/bbrc.1996.1081

[39] H. Macarthur, et al., "Selective Inhibition of Agonist-Induced but Not Shear Stress-Dependent Release of Endothelial Autacoids by Thapsigargin," British Journal of Pharmacology, Vol. 108, No. 1, 1993, pp. 100-105.

[40] S. N. Doshi, et al., "Flow-Mediated Dilatation Following Wrist and upper Arm Occlusion in Humans: The Contribution of Nitric Oxide," Clinical Science, Vol. 101, No. 6, 2001, pp. 629-635. doi:10.1042/CS20010033

[41] M. Lie, O. M. Sejersted and F. Kiil, "Local Regulation of Vascular cross Section during Changes in Femoral Arterial Blood Flow in Dogs," Circulation Research, Vol. 27, No. 5, 1970, pp. 727-737.

[42] S. M. Hilton, "A Peripheral Arterial Conducting Mechanism Underlying Dilatation of the Femoral Artery and Concerned in Functional Vasodilatation in Skeletal Muscle," The Journal of Physiology, Vol. 149, 1959, pp. 93-111.

[43] Y. Inaba, J. A. Chen and S. R. Bergmann, "Prediction of Future Cardiovascular Outcomes by Flow-Mediated Vasodilatation of Brachial Artery: A Meta-Analysis," International Journal of Cardiovascular Imaging, Vol. 26, 
No. 6, 2010, pp. 631-640.

doi:10.1007/s10554-010-9616-1

[44] D. J. Green, et al., "Flow-Mediated Dilation and Cardiovascular Event Prediction: Does Nitric Oxide Matter?"
Hypertension, Vol. 57, No. 3, 2011, pp. 363-9. doi:10.1161/HYPERTENSIONAHA.110.167015 